Article

\title{
Three-Stage Analysis of the Maximum Accommodation Capacity of a Distribution System with High Photovoltaic Penetration
}

\author{
Jiaqi Gu ${ }^{1}$, Fei Mei ${ }^{1, * \mathbb{C}}$, Jixiang $\mathrm{Lu}^{2}{ }^{2}$, Jinjun Lu ${ }^{2}$, Jingcheng Chen ${ }^{3}$, Xinmin Zhang ${ }^{3}$ and \\ Limin $\mathrm{Li}^{4}$ \\ 1 College of Energy and Electrical Engineering, Hohai University, Nanjing 211100, China; gujiaqi@hhu.edu.cn \\ 2 State Key Laboratory of Smart Grid Protection and Control, NARI Group Corporation, \\ Nanjing 211000, China; lujixiang@sgepri.sgcc.com.cn (J.L.); lujinjun@sgepri.sgcc.com.cn (J.L.) \\ 3 State Grid Tianjin Electric Power Company, Tianjin 300010, China; jingcheng.chen@tj.sgcc.com.cn (J.C.); \\ xinmin.zhang@tj.sgcc.com.cn (X.Z.) \\ 4 Tianjin Huadian Beichen Distributed Energy Co., Ltd., Tianjin 300400, China; limin-li@chd.com.cn \\ * Correspondence: meifei@hhu.edu.cn; Tel.: +86-152-9552-9785
}

Received: 24 July 2020; Accepted: 19 August 2020; Published: 20 August 2020

\begin{abstract}
The safety and stability of a distribution network will be affected by high photovoltaic (PV) penetration. Therefore, it is of great significance to evaluate the PV accommodation capacity of a distribution network and to select an appropriate PV accommodation scheme. This paper assesses the PV accommodation capacity of a distribution network with an improved algorithm and optimizes the accommodation scheme with a comprehensive index. First, the PSO (particle swarm optimization)-Monte Carlo algorithm is used to evaluate the maximum accommodation capacity of a distribution network with PV integration. Second, a year-round voltage timing simulation is performed to analyze the node voltage that exceeds the limit under the planned PV capacity, which is higher than the previously evaluated maximum accommodation capacity. Finally, the staged control strategy of the PV inverter and energy storage is carried out to select the scheme for the sizing and siting of energy storage. The simulation tests use a $10 \mathrm{kV}$ standard distribution network as an example for PV evaluation and PV accommodation scheme selection to verify the feasibility and effectiveness of the proposed model.
\end{abstract}

Keywords: PV power accommodation; PSO-Monte Carlo algorithm; the staged control strategy of PV inverter and energy storage; the sizing and siting of energy storage

\section{Introduction}

In recent years, renewable energy has been strongly promoted due to its advantages, such as cleanliness, large reserves and renewability [1,2]. The most typical example is photovoltaic (PV) energy [3].

Although large-scale PV power has access to distribution networks, reducing power loss [4] and providing voltage support [5], high PV penetration in a distribution network will impact the safety [6], stability [7] and economy [8] of the distribution system. Thus, it is of great significance to correctly assess the PV accommodation capacity and to select reasonable accommodation schemes for a PV-rich-distribution network.

To date, many countries have conducted extensive and far-reaching research on PV accommodation capacity assessment and methods. The evaluation of the accommodation capacity of a distribution network can be performed with the dynamic simulation method and mathematical optimization method. The dynamic simulation method is based on some dynamic simulation software $[9,10]$, such as 
OpenDss or PSCAD. The principle of this method is relatively simple but repeated, and a large number of calculations may be needed. The mathematical optimization method aims to solve the problem by the mathematical method, the intelligent algorithm method or the random scene simulation method [11-13]. Ayres processed the distribution network model linearly, and the capacity could be calculated by the voltage sensitivity matrix [14]. Koutroumpezis evaluated the maximum accommodation capacity of the distribution network through the optimization algorithm under the constraints of node voltage and line current, but the simulation scene was still a single scenario [15]. Furthermore, the Monte Carlo algorithm was adopted to randomly select the access points, access number and access capacity of a distribution PV system, and the PV accommodation capacity of the distribution network was obtained through a variety of scenario simulations [16]. However, the random scene simulation method requires a large number of calculations and takes a long time. Therefore, the evaluation algorithm can be further improved and modified.

Reasonable PV consumption methods can ensure the safety and stability of the distribution network $[17,18]$. Improving the PV accommodation capacity of a distribution network can be considered based on the power factor adjustment of PV inverters [19,20], energy storage technology [21,22], reactive power compensation $[23,24]$, and grid reconstruction $[25,26]$. With the adjustment of a PV inverter, node voltage was returned to the normal value through staged control of the inverter [27], but the author did not consider the situation from the planned perspective. Zhao used the $\mathrm{L}$ index to determine the sizing and siting of energy storage [28]. Due to the correlation between nodes, the single-index configuration of energy storage is not the best choice. Tao built a bi-level planning model to configure the siting and sizing of energy storage [29]. However, the bi-level planning model is relatively complex, and the upper layer addresses energy storage at random. Taking the correlation between nodes into consideration, the distribution network is divided into several clusters [30]. According to the results of cluster division, a bi-level planning model was established to optimize the siting and sizing of installation PV and energy storage.

Considering some problems in terms of PV consumption method and evaluation, this paper uses a three-stage analysis of maximum accommodation capacity, completing the evaluation of PV accommodation capacity, the formulation of a PV accommodation scheme and the selection of a PV accommodation scheme in three stages. First, the PSO-Monte Carlo algorithm is used to evaluate the PV accommodation capacity with regards to calculation speed and calculation accuracy. Second, according to the results of the evaluation stage, a year-round voltage timing simulation is carried out from a planned perspective. The staged control strategy of the energy storage and inverter is carried out to formulate schemes of the sizing and siting of the energy storage in terms of the node voltage that exceeds the limit. Finally, the average voltage fluctuation index and maximum accommodation capacity index are introduced into each scheme. GRA (grey correlation analysis) and AHP (analytic hierarchy process) are used to choose the suitable scheme from the schemes in the stage of scheme formulation. Thus, the main contributions of the paper are summarized as follows:

- Compared to traditional algorithms, PSO is used to optimize the Monte Carlo algorithm, which not only improves the calculation speed, but also improves the calculation accuracy in this paper.

- Considering the characteristics of the correlation between nodes, two indexes are introduced to determine the sitting schemes of the energy storage devices objectively. Additionally, a cooperative game theory is proposed to optimize energy storage capacity and the annual abandoned PV power.

- This article considers the impact of energy storage and abandoned PV power on the distribution network, and proposes the average improvement index of node voltage fluctuation and a maximum PV accommodation capacity index. Scheme selection is based on energy storage capacity, abandoned PV power and their influencing factors instead of simply considering energy storage capacity and abandoned PV power. 


\section{Three-Stage Analysis Process of PV Accommodation}

This article mainly starts from the perspective of PV evaluation and the consumption method. We will divide this article into three stages. The first stage is mainly used to evaluate the PV accommodation capacity of the distribution network, while the second and third stages mainly carry out the work of PV consumption. Because the energy storage devices are used, it is necessary to consider the siting and sizing of the energy storage devices. Therefore, the schemes of the siting of the energy storage device are formulated in the second stage, and the energy storage capacity and annual total abandoned PV power are also determined. The third stage mainly explores the impact of energy storage and abandoned PV power on the distribution network, and select the final scheme with their influencing factors. Therefore, the three stages are PV accommodation capacity assessment, PV accommodation scheme formulation, and PV accommodation scheme selection, respectively. The specific measures in each stage are as follows:

The first stage: The PSO-Monte Carlo algorithm is used to assess the PV accommodation capacity of a distribution network with high calculation speed and accuracy.

The second stage: The PV capacity configuration is carried out from the planned perspective according to the result of the evaluation in the first stage. A year-round voltage timing simulation is performed to analyze the node voltage that exceeds the limit. The staged control strategy of the inverter and energy storage is adopted to formulate the different schemes of the sizing and siting of the energy storage. In this stage, the cluster strategy and the degree of node voltage that exceeds the limit are used to determine the siting of energy storage. The cooperative game algorithm is used to optimize the capacity of energy storage and abandoned PV power.

The third stage: The average improvement of node voltage fluctuation and maximum accommodation capacity indexes are introduced. Combining the four indicators of each scheme, the AHP and GRA are used to select the most appropriate scheme and finish determining the sizing and siting of energy storage.

\section{PV Accommodation Capacity Assessment}

\subsection{Typical Moment}

The permeability of the PV is defined as Equation (1).

$$
\lambda_{p v}(t)=\frac{P_{p v}(t)}{P_{\text {load }}(t)}
$$

where $\lambda_{p v}(t)$ is PV permeability at the time $t, P_{p v}(t)$ is the PV power at the time $t, P_{\text {load }}(t)$ is the load power at the time $t$, and $t$ is one hour of annual hours from 1 to 8760 .

In order to avoid the effect of different types of power, the annual load power and PV power are normalized to obtain the load annual fluctuation coefficient and PV annual fluctuation coefficient. Additionally, the annual fluctuation coefficient of load power and PV power is used to pick out the typical moment in Equation (2), which is reasonably verified in [31].

$$
I_{p v}(t)=\frac{P_{p v}(t) / P_{p v, \max }}{P_{\text {load }}(t) / P_{\text {load, } \max }}
$$

where $P_{p v, \text { max }}$ is the maximum PV power throughout the year, and $P_{l o a d, \max }$ is the maximum load power throughout the year.

Figure 1 shows the process of annual load power and PV power fluctuation, which correspond to the denominator and numerator of Equation (2), respectively. According to Equation (2), the typical moment is $3399 \mathrm{~h}$ when the PV power is 0.7212 times smaller than the maximum PV power and the load power is 0.1811 times smaller than the maximum load power. At this time, the selected typical 
moment is not at the moment of minimum load or the maximum PV power but at the moment when the load is relatively low and the PV power is relatively high.

It is also the most appropriate time to explore the maximum accommodation capacity of a distribution network under this moment.

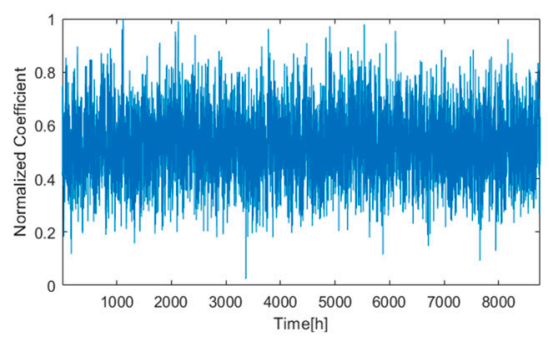

(a)

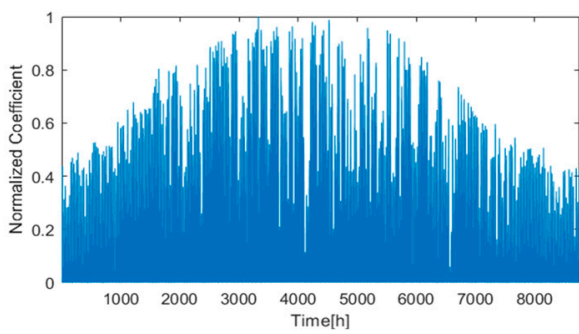

(b)

Figure 1. (a) Load annual fluctuation coefficient; (b) photovoltaic annual fluctuation coefficient.

\subsection{PSO-Monte Carlo algorithm}

Although Monte Carlo can accurately evaluate the accommodation capacity of a distribution network, the accuracy is related to the number of scene simulations. The longer time it takes, the more accurately it assesses the capacity. In terms of the optimization algorithm, it is easy to fall into a local optimum if the initial value is not properly selected.

Considering the advantages and disadvantages of the algorithms, this paper uses the PSO to optimize the Monte Carlo algorithm to evaluate the accommodation capacity of a distribution system more accurately and quickly.

First, Monte Carlo is used to preliminarily obtain the maximum PV accommodation capacity and the PV capacity configuration of each node. Then, the PV capacity of each node is used as a reference to optimize initial value of PSO for the optimal solution. The process of evaluating PV accommodation capacity of a distribution network with the PSO-Monte Carlo algorithm is shown in Figure 2. The specific steps are as follows:

1. Determination of the number of PV grid connections: $N$ accessible PV nodes can be connected to the distribution system from the load nodes of the distribution network.

2. Selection of PV grid connection location: $N_{p v}$ PV nodes can be selected and connected to the distribution system from $N$ accessible PV nodes.

3. Configuration of PV grid-connected capacity: The capacity of the accessible PV node is calculated based on $\lambda_{p v}$ of its node power in Equation (3).

$$
C_{p v}=\lambda_{p v} P_{l o a d}
$$

4. Determination of accessible PV power: According to the normalized PV fluctuation coefficient and local temperature, the PV power is calculated in combination with the PV grid connection capacity.

5. Power flow calculation: The forward and backward substitution method is used to calculate the voltage and power of each node according to the PV power, load power and distribution network parameters.

6. Repeated calculation: $\lambda_{p v}$ is increased step by step until it reaches the preset maximum value. The maximum PV accommodation capacity of the distribution system is evaluated, and the PV installation capacity of each node is obtained under voltage safety constraints.

7. The maximum accommodation capacity evaluation: The PV capacity value of the PSO algorithm in each node is initialized according to the PV installation capacity of each node in step 6. The maximum accommodation capacity is assessed through the PSO optimization operation. 


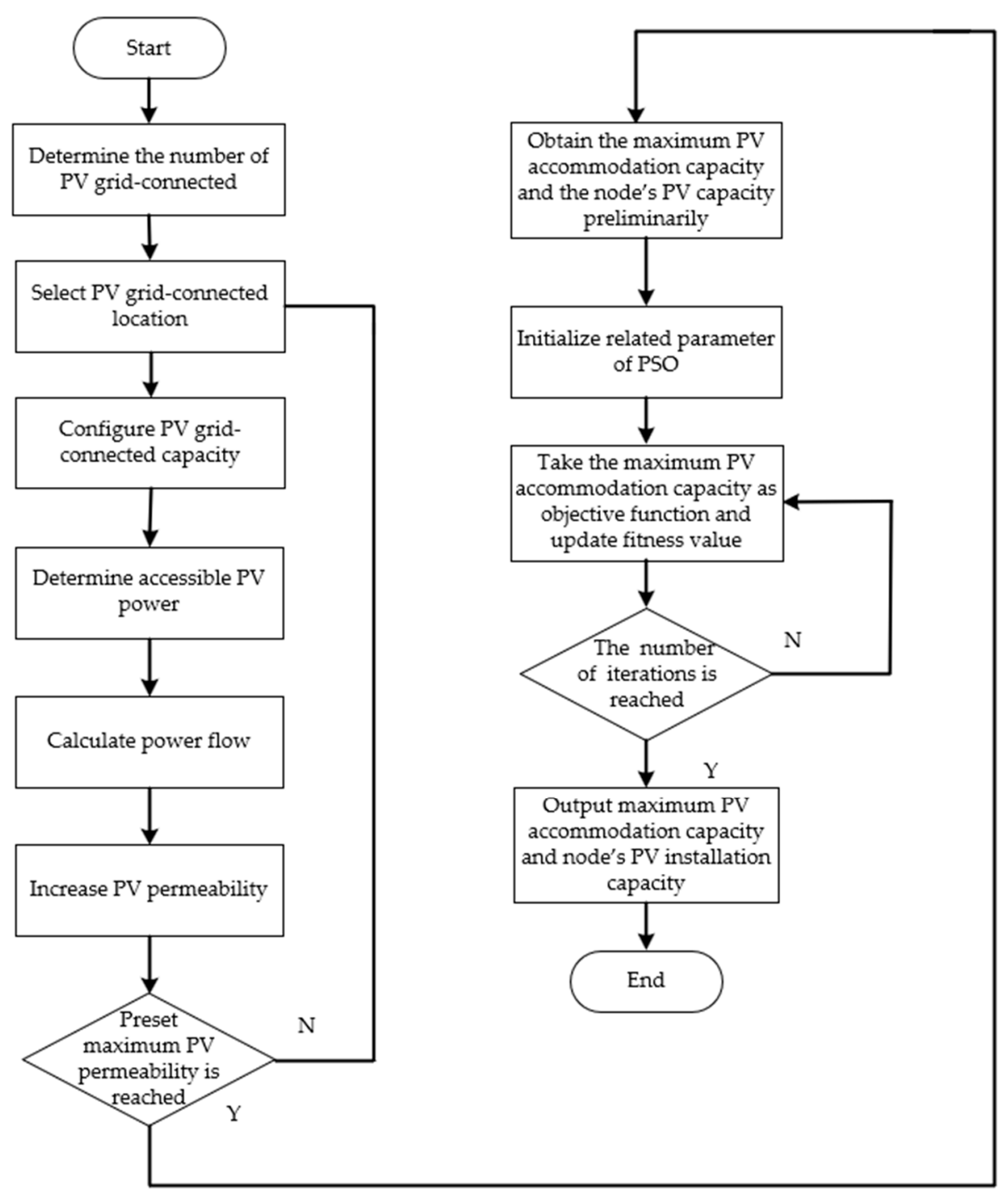

Figure 2. Evaluation process with the PSO-optimized Monte Carlo algorithm.

\section{PV Accommodation Scheme Formulation}

\subsection{The Process of PV Accommodation Scheme Formulation}

When the maximum evaluation does not meet the demand of the planned PV capacity of the distribution network, it is necessary to study the annual timing condition of node voltage under the planned capacity. According to the PV capacity configured by each node in the evaluation stage, the PV capacity of each node will increase in proportion to the planned capacity, and the year-round voltage timing simulation will proceed. The staged control strategy of inverter and energy storage is adopted to carry out the PV accommodation, the process of which is shown in Figure 3. The specific measures are as follows:

1. Annual voltage timing simulation from the planned perspective is carried out.

2. According to the condition of node voltage off-limit, the inverter reactive power regulation is used to carry out preliminary adjustment on the node, and the node voltage that exceeds the limit can be obtained after adjustment.

3. According to the degree to which the node voltage exceeds the limit and cluster strategy, the siting schemes of energy storage are formulated. 
4. For different siting selection schemes, cooperative games are used to optimize the power of nodes configured energy storage. The optimal energy storage configuration capacity and the total annual abandoned PV power are obtained by cooperative games. Active PV power is discarded only at the node where the PV equipment is installed. In addition, the rate of total annual abandoned PV power is required to be less than $0.01 \%$.

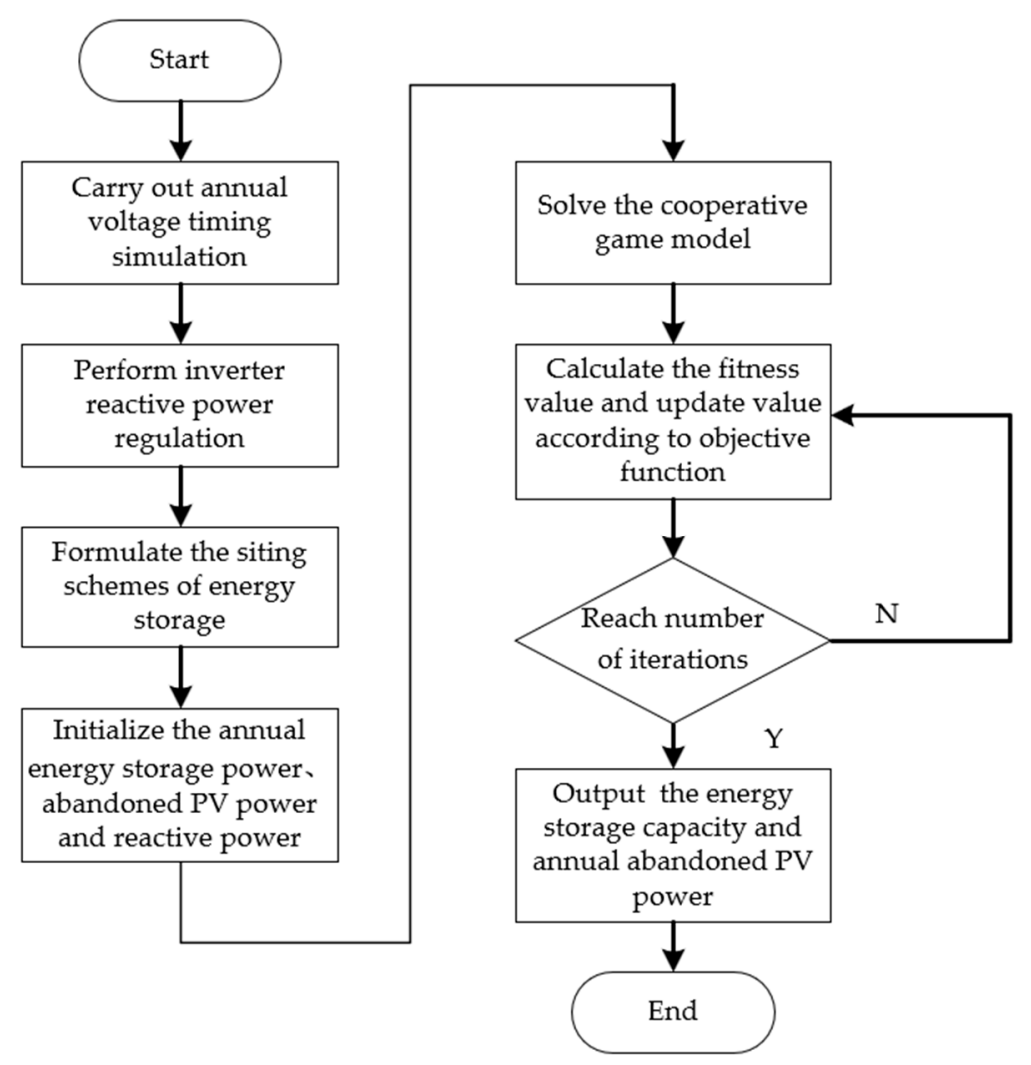

Figure 3. The process of the PV accommodation scheme formulation.

\subsection{Staged Control Strategy of the PV Inverter and Energy Storage}

The voltage regulation of the PV inverter can be roughly divided into two stages. The first stage is the regulation of the inverter reactive power until the maximum power factor or the maximum inverter capacity is reached. This stage will not discard the PV active power. The second stage is the regulation of the active power of the inverter. Discarding active power will be carried out at this stage. The energy storage stage is the same as the second stage of the inverter, which is adjusting the active power. Therefore, the inverter's reactive power adjustment can be used to perform the first round of voltage adjustment, and energy storage combined with abandoned PV power are used as the second stage of adjustment if the node voltage exceeds the limit value.

\subsection{The Siting of the Energy Storage}

According to the abovementioned staged control strategy, the problems of the sizing and siting of energy storage need to be considered when using the energy storage. To solve these problems, a bi-level planning model is used to find the siting and sizing of the energy storage. The upper layer is for the siting of energy storage, and the lower layer is for the sizing of energy storage. The bi-level planning model has a certain complexity, and the upper layer uses random particles to find the position, which is a blinded method. Aiming at the shortcomings of bi-level planning, this paper will combine the degree to which the node voltage exceeds the limit and cluster strategy to conduct a comprehensive evaluation in order to formulate the energy storage siting scheme. 


\subsubsection{Cluster Strategy}

Considering the strong and weak associations between nodes, the cluster strategy is used to divide the strong and weak node associations. The correlation between clusters is relatively weak, and the correlation within clusters is relatively strong. In this paper, the cluster division adopts the division method of [30]. The electrical distance is used as a structural index to measure the degree of the correlation of the changes in node voltage amplitude; the power balance is used as a power index to measure the effect of active and reactive power changes on the node.

\subsubsection{Node Voltage Out-Of-Limit Degree}

The indicator of the degree to which the node voltage exceeds the limit is used to measure the magnitude to which the node voltage is higher than the safety threshold at the moment when the voltage exceeds the limit, which is shown in Equation (4).

$$
D_{i}=\sum_{t=1}^{M}\left|U_{i, t}-U_{N \max }\right|
$$

where $M$ is the number of hours when the voltage exceeds the limit in a year, $U_{N \max }$ is the upper limit value of safety voltage constraint, and $U_{i, t}$ is voltage of node $i$ at the time $t$.

\subsection{The Sizing of the Energy Storage}

With the energy storage siting decided, a cooperative game is used to select the energy storage configuration in order to optimize the energy storage configuration capacity. At the same time, to reduce the energy storage capacity configuration and reduce the energy storage cost, part of the PV power is abandoned when the voltage exceeds the limit.

\subsubsection{Cooperative Game Model}

Cooperative game refers to the game behavior of all participants who are collectively rational and coordinate with each other to determine their own strategies. The game results are beneficial to all the participants. In this paper, the elements of participation in the cooperative game model are as shown below:

1. Participants: $N_{C}$ nodes installed in the energy storage system.

2. Strategy set: the annual charge and discharge power of energy storage, annual discarded PV power and reactive power at every moment at different nodes.

3. Characteristic function: the sum of energy storage capacity of the nodes and the annual abandoned PV power are the lowest in Equation (5).

$$
S_{t o l}=\sum_{n=1}^{N_{C}} \sum_{t=1}^{T} S_{n}\left(P_{c h, t}, P_{\text {dich }, t}, P_{g p, t}, Q_{t}\right)
$$

where $N_{C}$ is the number of nodes installed for energy storage, $S_{n}$ is sum of energy storage capacity and the annual abandoned PV power of node $n$, and $Q_{t}$ is the reactive power at the time $t$ of node $n$.

\subsubsection{The Constraint of the Model}

The use of energy storage and abandoned PV power for annual node scheduling optimization needs to satisfy the state-of-charge constraints of energy storage, charge-discharge constraints of energy storage, and abandoned PV power constraints.

1. Energy storage charging and discharging power: 
Power charging and discharging cannot occur simultaneously, as is shown in Equation (6).

$$
P_{c h, t} \cdot P_{d i s, t}=0
$$

where $P_{c h, t}$ is the charging power of energy storage at time $t$, and $P_{d i s, t}$ is the discharging power at time $t$.

2. State of charge of energy storage:

To prolong the life of the electricity storage equipment, it is necessary to ensure that the state of charge of the electricity storage equipment is within a certain range.

$$
\begin{gathered}
S_{e, t}=S_{e, t-1}+\frac{P_{c h, t} \eta_{e c h} \Delta t}{S_{N e}}-\frac{P_{d i s, t} \Delta t}{S_{N e} \eta_{e d i s}} \\
S_{e \min } \leq S_{e, t} \leq S_{e \max }
\end{gathered}
$$

where $S_{e, t}$ is the state of charge at the time $t, S_{e, t-1}$ is the state of charge at the time $t-1, \eta_{e c h}$ is the charging efficiency of the energy storage device, $\eta_{\text {edis }}$ is the discharging efficiency of the energy storage device, $S_{N e}$ is the rated capacity of the energy storage device, and $\Delta t$ is the unit scheduling period.

3. Abandoned PV power constraint:

The abandoned PV power needs to be less than the PV power generated at a certain moment.

$$
P_{g p, t} \leq P_{p v, t}
$$

where $P_{g p, t}$ is the abandoned PV power at the time $t$, and $P_{p v, t}$ is the PV power at the time $t$.

\section{PV Accommodation Scheme Selection}

Since the total energy storage configuration capacity and abandoned PV power of different nodes in different schemes are different, it is impossible to objectively measure the quality of different schemes by simply relying on energy storage configuration and abandoned PV power. Therefore, to balance and objectively measure the energy storage configuration and the abandoned PV power in each scheme, an average improvement indicator of node voltage fluctuation and a maximum consumption capacity indicator are introduced. For the four indicators in each plan, the final choice of the plan is to use a combination of AHP and GRA. Figure 4 shows the process of PV accommodation scheme selection, and the specific steps are as follows:

1. An average improvement of node voltage fluctuation and maximum accommodation capacity are calculated in each scheme.

2. A numerical matrix of four indicators in different schemes is built.

3. The numerical matrix is normalized as a sample sequence and the optimal reference sequence is selected.

4. The grey correlation is calculated according to the relationship between the optimal reference sequence and sample sequence.

5. AHP is used to determine the weight of GRA.

6. Weighted relevance, representing the degree of association with the optimal sample sequence, is calculated through weight and grey correlation.

7. The best scheme according to weighted relevance is sorted and chosen. 


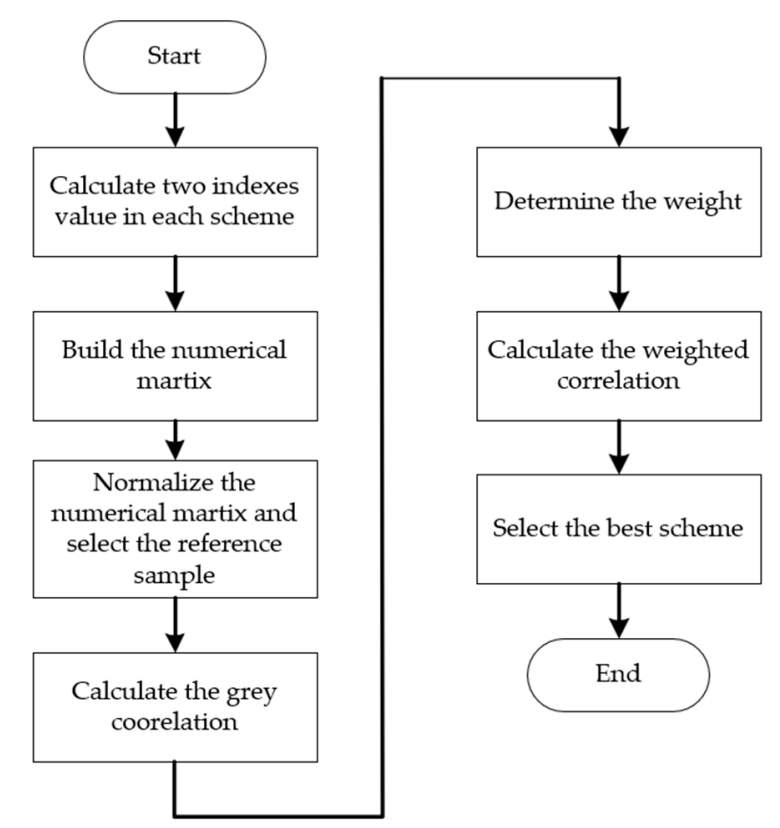

Figure 4. The process of photovoltaic accommodation scheme selection.

\subsection{Index Introduction}

To more objectively observe the impact of energy storage and abandoned PV power on the distribution network, two new indexes will be introduced.

\subsubsection{The Average Improvement Indicator of Node Voltage Fluctuation}

The energy storage device absorbs the PV power when the PV power is greater than the load power and releases the power when it is less than the load power. Therefore, it can improve the node voltage, and its voltage fluctuation improvement index can be defined in Equation (10).

$$
\begin{gathered}
d u_{i}=\frac{d U_{i}-d U_{i}^{\prime}}{d U_{i}} \\
d U_{i}=\sum_{t=1}^{T}\left|U_{i, t}-U_{i, a v}\right| \\
d U_{i}^{\prime}=\sum_{t=1}^{T}\left|U_{i, t}^{\prime}-U_{i, a v}^{\prime}\right|
\end{gathered}
$$

where $U_{i, t}$ and $U_{i, t}^{\prime}$ are the voltage before and after improvement of the node $i$ at the time $t$, respectively, $U_{i, a v}$ and $U_{i, a v}^{\prime}$ are the annual average voltages of node $i$ before energy storage installation and after energy storage installation, respectively; and $d U_{i}$ and $d U_{i}^{\prime}$ are the voltage fluctuations of the node $i$ before energy storage installation and after energy storage installation, respectively.

Since the number of energy storage configuration nodes is different under different schemes, the average fluctuation index is used for measurement in Equation (11).

$$
M_{\text {ind }}=\frac{1}{N_{C}} \sum_{i=1}^{N_{C}} d u_{i}
$$

\subsubsection{Maximum Accommodation Capacity Index}

Through energy storage and abandoned PV power processing, the node voltage can be restored to normal, and the node voltage level can be improved. The energy storage configuration of different schemes and the situation of discarding PV power are also different for the improvement of the new round of accommodation capacity. 
The evaluation of the new maximum accommodation capacity is measured under the energy storage capacity, energy storage power, and annual PV power after the cooperative game. During the process of evaluating new maximum accommodation capacity, it is necessary to ensure that the charge and discharge of the energy storage at each moment do not exceed the energy storage charge and discharge power in the plan. The energy storage capacity and the abandoned PV capacity of the node configuration in the stage of new evaluation are consistent with the energy storage configuration and the total amount of abandoned PV power in the plan. In other words, the new round of accommodation capacity must be based on the node energy storage capacity, energy storage power and total annual abandoned PV power in the stage of the cooperative game.

\subsection{Scheme Evaluation}

For the four indicators of the above schemes, how to choose the most suitable scheme not only needs to take into account the correlation between the indexes and the ranking of the importance between the indexes, but it also needs to consider the data changes of different schemes within the indexes. Therefore, the correct choice of a better plan requires a combination of subjective and objective ways to choose a reasonable plan. AHP is a combination of quantitative methods and qualitative methods, using the experience of decision makers to judge the relative importance of the standards that measure whether the goals are achieved, and giving a reasonable weight for each standard of each decision-making plan. GRA is a multi-factor statistical analysis method, which is based on the sample data of each factor and uses the grey correlation degree to describe the strength, size and order of the relationship between the factors. That is, AHP focuses more on subjective experience, weights of which are used to find the important order of index, while GRA focuses more on the numerical relationship of data factors. In this paper, GRA is used to analyze the degree of correlation between the values in different indexes. AHP is used to determine the importance of different indexes according to subjective judgement. The specific evaluation process with AHP and GRA is as follows:

1. Determine the sample and optimized reference sequence:

There are a total of $m$ samples in $n$ indicators composing the numerical matrix $X=\left(x_{i j}\right)_{m n}$. The numerical matrix is normalized as the sample sequence with Equation (12), and the optimized reference sequence is selected from the sample sequence with Equation (13). The optimized reference sequence is optimal value in $n$ indicators, which is the minimum or maximum value in $n$ indicators.

$$
\begin{gathered}
c_{i j}=\frac{x_{i j}-\min _{j} x_{i j}}{\max _{j} x_{i j}-\min _{j} x_{i j}} \\
d_{j}=\operatorname{opt}\left(c_{i j}\right)
\end{gathered}
$$

where $x_{i j}$ is the corresponding value in $i$ samples of $j$ indicators, $i=1,2, \cdots, m, j=1,2, \cdots, n$, and $d_{j}$ is the optimal value in the $j$ indicator.

2. Calculate the grey correlation:

$\Delta_{i j}$ is defined as the absolute deviation between the $i$ value sample and optimal value sample in the $j$ indicator in Equation (14).

$$
\Delta_{i j}=\left|c_{i j}-d_{j}\right|
$$

The grey correlation $\varepsilon_{i j}$ can be calculated by Equation (15).

$$
\varepsilon_{i j}=\frac{\min _{i} \min _{j} \Delta_{i j}+\rho \max _{i} \max _{j} \Delta_{i j}}{\Delta_{i j}+\rho \max _{i} \max _{j} \Delta_{i j}}
$$


The general resolution coefficient value $\rho$ is 0.5 , which lacks objectivity. The resolution coefficient $\rho$ is determined according to reference [32], which makes it more consistent with the sample data and more objective.

3. Determine the weight of GRA with AHP:

A judgement matrix combining the subjective importance of the indexes is constructed. The judgement matrix must pass the consistency test. Weight $\omega=\left(\omega_{1}, \omega_{2}, \cdots, \omega_{j}\right)$ can be obtained after meeting the AHP standard principle.

4. Calculate weighted relevance:

Weighted relevance can be calculated with Equation (16), which reflects the correlation between the optimal sample and value sample.

$$
\gamma_{i}=\sum_{j=1}^{n} \varepsilon_{i j} \omega_{j}
$$

5. Select the final scheme:

The greater the value of $\gamma_{i}$, the greater the correlation between the first sample and the optimal sample. Therefore, the optimal solution can be selected according to the degree of correlation.

\section{Analysis of Examples}

\subsection{Parameters of Examples}

This example uses MATLAB2019a for the simulation. The operating system is a 64-bit Windows 10 system, the RAM is $4.0 \mathrm{~GB}$, the CPU is Intel Core I7-5500U, $2.4 \mathrm{GHz}$, and the core frequency is 3.0 GHz. This example uses the IEEE 33 system, which is shown in Figure 5. The reference voltage of the system is $12.66 \mathrm{kV}$. The base power of the system is $10 \mathrm{MVA}$. Node 1 is the balanced node whose voltage is $1.04 \mathrm{pu}$, and the total system load is $3.72 \mathrm{MW}+\mathrm{j} 2.30$ Mvar. The PV output system and load fluctuation coefficient are shown in Figure 1 above, and the ambient temperature is taken as $25^{\circ} \mathrm{C}$. The inverter limit power factor is set to 0.95 . To ensure that the inverter has sufficient reactive power regulation capacity, the inverter capacity is set to 1.1 times higher than the PV installation capacity. The unit period for optimized scheduling is $1 \mathrm{~h}$, and the total period for optimized scheduling is 1 year.

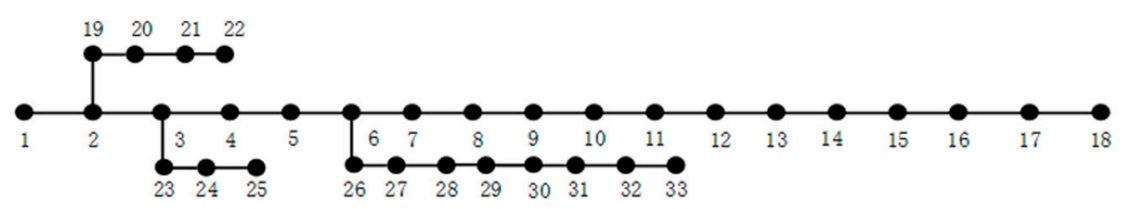

Figure 5. Standard IEEE 33 node distribution network model.

Considering that the urban area of the distribution network is relatively small, it is considered that the change in light density within a distribution network is not very large, so the light intensity of each node is considered to be the same.

This paper will take node voltage as the constraint and meet equal constraints of active and reactive power in the distribution network. Considering the assessment standards of power supply companies, the maximum safety unit value of the node voltage shall not exceed $1.05 \mathrm{pu}$, and the minimum safety unit value shall not be less than $0.95 \mathrm{pu}$.

\subsection{Evaluation of the Results of Accommodation}

To objectively evaluate the effect of the PSO-Monte Carlo algorithm, this paper compares the calculation time and accuracy between the traditional Monte Carlo algorithm and the optimized Monte Carlo algorithm. $\lambda_{p v}$ can be adopted from 0.1 to 3 . The interval step increment is 0.1. 
The traditional algorithm will run 2000 times to simulate scenes with each increase in step, that is, a total of 60,000 times using the Monte Carlo algorithm for random scene simulation.

The optimized algorithm will run 200 times to simulate scenes with each increase in step, that is, a total of 6000 times using the PSO-Monte Carlo algorithm for random scene simulation.

Related parameters of the PSO algorithm are set as the upper and lower limits to avoid local optimal solution, and the value of three parameters will be changed during each iteration in Equation (17).

$$
\begin{aligned}
& \mathrm{W}=\mathrm{W}_{\max }-\frac{\left(\mathrm{W}_{\max }-\mathrm{W}_{\min }\right) k}{C_{\text {Maxiter }}} \\
& C_{1}=C_{1 \max }-\frac{\left(C_{1 \max }-C_{\min }\right) k}{C_{\text {Maxiter }}} \\
& C_{2}=C_{2 \max }-\frac{\left(C_{2 \max }-C_{2 \min }\right) k}{C_{\text {Maxiter }}}
\end{aligned}
$$

where $\mathrm{W}$ is inertia weight, $\mathrm{W}_{\max }$ and $\mathrm{W}_{\max }$ are the upper and lower limits of $\mathrm{W}, \mathrm{C}_{1}$ and $C_{2}$ are learning factors, $C_{1 \max }$ and $C_{1 \min }$ are the upper and lower limits of $C_{1}, C_{2 \max }$ and $C_{2 \min }$ are the upper and lower limits of $C_{2}, k$ is the number of current iteration, and $C_{\text {Maxiter }}$ is the number of the maximum iteration.

A large number of experimental simulations is conducted to ensure the global optimal solution. $\mathrm{W}_{\max }$ and $\mathrm{W}_{\min }$ are set to 0.5 and 0 , respectively. $C_{1 \max }$ and $C_{1 \min }$ are set to 2 and 1 , respectively. $C_{2 \max }$ and $C_{2 \min }$ are set to 2 and 1, respectively. $C_{\text {Maxiter }}$ is 500 .

The results are shown in Table 1.

Table 1. Results of different algorithms.

\begin{tabular}{ccc}
\hline Algorithm & Time/s & Maximum Accommodation Capacity/MW \\
\hline Monte Carlo & 177.80 & 3.65 \\
PSO-Monte Carlo & 3.07 & 4.53 \\
\hline
\end{tabular}

Table 1 shows that the PSO-Monte Carlo algorithm exhibits a certain improvement in terms of calculation time and accuracy.

The first step uses Monte Carlo to simulate the scene to obtain the maximum PV accommodation capacity of the distribution network and the PV capacity of the nodes. The node configuring the PV system is not a weak voltage node but a strong voltage support node that can accommodate higher PV capacity. However, there is a problem that different voltage support nodes are also different for the capacity of accommodating PV. Since the PV capacity at the node in the simulation scenes increases in proportion to the load power of the node, the capacity evaluation is stopped until the minimum accommodation capacity of the voltage support point is reached. If the maximum accommodation capacity is assessed as much as possible, the voltage support node of the maximum accommodation capacity should be configured first. The aim of PSO is usually to target the maximum capacity and optimize the PV capacity at the voltage support node. This approach can avoid the limitation of the minimum consumption node and obtain more accurate results of the assessment of the consumption. If the Monte Carlo algorithm is not used to find the strong voltage support nodes and the initial value of PSO, PSO can easily fall into a local optimum. Thus, the accommodation capacity evaluation is higher than the single Monte Carlo algorithm and the PSO algorithm.

The accuracy of the Monte Carlo algorithm lies in the simulation of a large number of scenes. In this paper, 60,000 scenes are used for simulation to obtain the PV accommodation capacity under this algorithm. However, a large number of scene simulations affect the calculation time. The more simulation scenes there are, the longer time it takes, whereas when using the PSO algorithm to optimize the Monte Carlo algorithm, the number of simulation scene is reduced by 10 times, which greatly reduces the calculation speed. In addition, although number of iterations of the PSO is set to 500, the calculation time is relatively small after testing. Therefore, the overall calculation speed of the improved algorithm is much faster than the traditional algorithm. 


\subsection{The Result of a Year-Round Voltage Timing Simulation}

According to the distribution of the node PV capacity in Figure 6, the capacity of some nodes is almost 0 , the PV installation capacity of some nodes is less than $100 \mathrm{~kW}$, and the capacity of some nodes is higher than $100 \mathrm{~kW}$. It is worth noting that the boundary of this simulation is only a simulation of the scene with the largest installation capacity.

It is assumed that when the planned PV capacity of the distribution network reaches $6 \mathrm{MW}$, the original node PV access capacity increases in proportion. According to the annual PV factor and the load factor, the annual voltage timing simulation is carried out. Since node 24 is installed with the largest PV capacity, the node voltage limit is also the most serious, as shown in Figure 7. Therefore, it is necessary to use the staged control strategy of the inverter and energy storage to restore the node to the specified value in order to ensure the safety and stability of the distribution network.

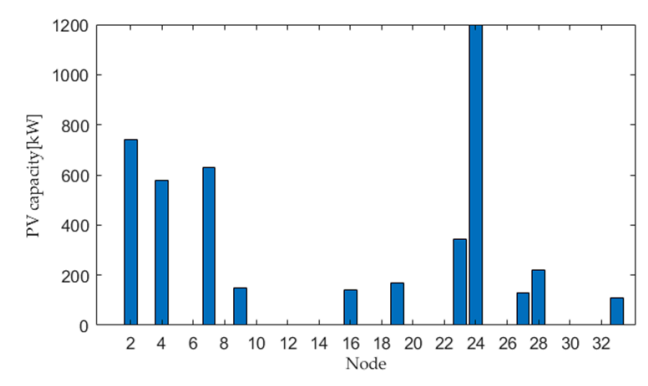

Figure 6. The distribution of node PV capacity.

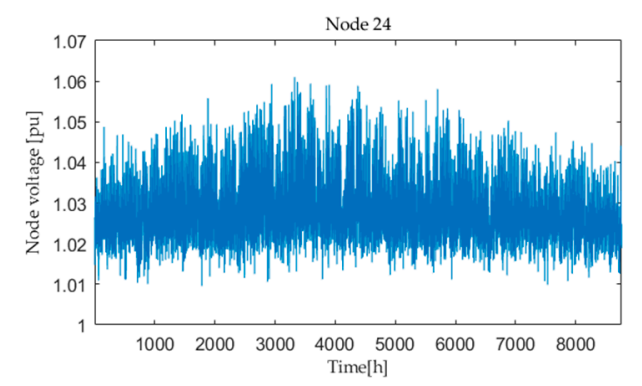

Figure 7. The annual voltage of node 24 under the planned capacity.

\subsection{The Siting of Energy Storage}

According to the voltage situation of each node simulated throughout the year, the selection of the energy storage siting scheme is combined with the degree to which the voltage exceeds the limit and cluster division strategy.

This paper divides the standard distribution network into four clusters through the principle of electrical distance and power balance under configuring the node PV capacity. The degree to which the voltage exceeds the limit for each node in each cluster is shown in Figure 8.

Figure 8 a shows that the nodes in cluster 1 that exceed the voltage limit are 23, 24, and 25 . There are no nodes in cluster 2 that exceed the voltage limit, so it is empty in Figure $8 \mathrm{~b}$. Figure $8 \mathrm{c}$ shows that the nodes in cluster 3 that exceed the voltage limit are 5, 6, 7, 8, 9, 26, 27, 28, 29, 30, and 33. Figure 8d shows that the voltage-over-limit nodes in cluster 4 are 10,11, and 12.

Considering the characteristics of the cluster, a preliminary selection is first made according to the degree to which the node voltage exceeds the limit in the cluster and, then, a comprehensive evaluation is conducted in combination with the relationship between the clusters. If the differences in the degrees to which the voltage exceeds the limit between adjacent nodes in the cluster is relatively large, the node with a severe voltage limit is considered to drive the node with a severely small voltage limit. Therefore, the voltage of the node with a lower voltage limit will naturally be dropped into the normal value when the voltage of node with a severe limit can be dropped into the specific value under the adjustment of 
the energy storage. After choosing the nodes in the cluster, we consider the correlation between the clusters. If the degree to which the maximum voltage limit in the cluster is much smaller than the degree to which the minimum voltage exceeds the limit in the remaining clusters, it is considered that the cluster voltage limit is driven by the remaining clusters. Thus, the energy storage will be installed in any node in this cluster.

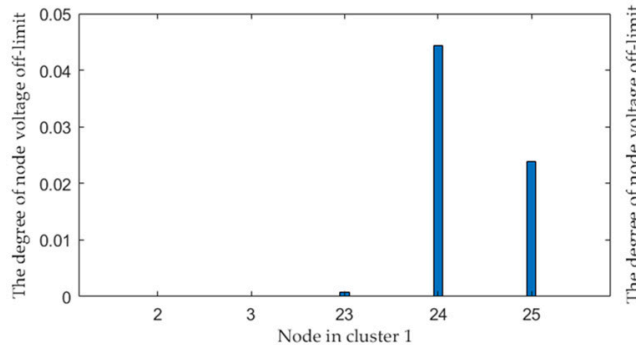

(a)

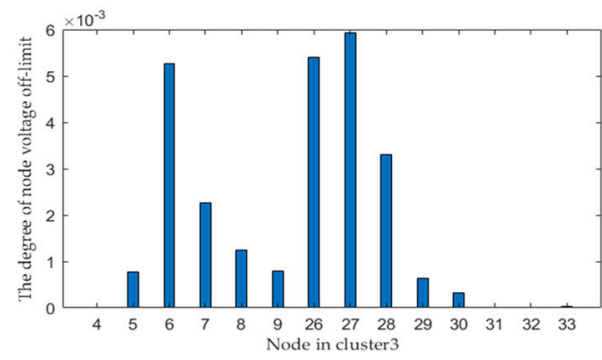

(c)

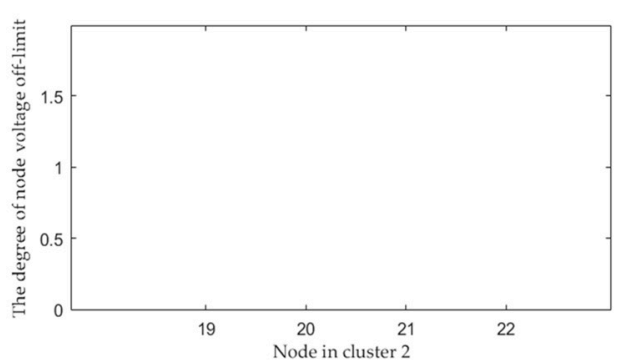

(b)

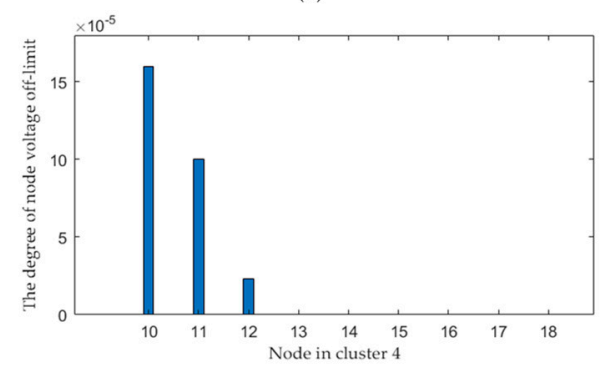

(d)

Figure 8. (a) The degree to which each node voltage exceeds the limit in cluster 1; (b) the degree to which each node voltage exceeds the limit in cluster 2; (c) the degree to which each node voltage exceeds the limit in cluster 3 ; (d) the degree to which each node voltage exceeds the limit in cluster 4.

In cluster 1, the degree to which the voltage exceeds the limit in nodes 24 and 25 is relatively large, while the degree to which the voltage exceeds the limit in node 23 is relatively small. It can be considered that the voltage of node 23 that exceeds the limit is driven by nodes 24 and 25 . Therefore, nodes 24 and 25 will be configured as energy storage. Cluster 2 has no voltage overrun, so it is not considered. The voltages of nodes $6,26,27$, and 28 in cluster 3 that exceed the limit are different from those of the remaining nodes. Therefore, the energy storage devices need to be installed. The degree to which the voltage exceeds the limit in cluster 4 is the lowest and significantly smaller than the remaining clusters. Therefore, it can be considered that the node voltage of cluster 4 that exceeds the limit is driven by the remaining clusters. Therefore, cluster 4 does not perform the energy storage configuration.

According to the abovementioned node energy storage configuration, the proposed nodes 24 , $25,6,27,26$, and 28 are used as candidate nodes of the energy storage configuration. The following energy storage configuration plan is drawn according to the degree to which the limit is exceeded in the clusters, as shown in Table 2.

Table 2. The siting scheme of energy storage.

\begin{tabular}{cc}
\hline Schemes & Node Configuring Energy Storage \\
\hline 1 & 24 \\
2 & 24,25 \\
3 & $24,25,27$ \\
4 & $24,25,27,26$ \\
5 & $24,25,27,26,6$ \\
6 & $24,25,27,26,6,28$ \\
\hline
\end{tabular}




\subsection{The Sizing of Energy Storage}

According to the above energy storage scheme, a cooperative game is performed to obtain the optimal energy storage capacity and the annual abandoned PV power. The result of specific energy storage capacity and rated power of each node in each scheme is shown in Table 3. Combining the two new indexes introduced, total energy storage capacity, annual total abandoned PV power, the average improvement of node voltage fluctuation and maximum PV accommodation capacity are calculated in each scheme, as shown in Table 4.

Table 3. The rated energy storage capacity and power of nodes in each scheme.

\begin{tabular}{|c|c|c|c|c|c|c|c|c|}
\hline & & Schemes & 1 & 2 & 3 & 4 & 5 & 6 \\
\hline \multirow{12}{*}{ Node } & \multirow{2}{*}{24} & Energy storage Capacity/kW·h & 3900 & 4270 & 4640 & 4640 & 4810 & 4680 \\
\hline & & Energy storage power/kW & 695 & 1230 & 1190 & 1180 & 1220 & 1180 \\
\hline & \multirow{2}{*}{25} & Energy storage Capacity/kW·h & 0 & 1780 & 2030 & 1960 & 1890 & 2020 \\
\hline & & Energy storage power/kW & 0 & 197 & 201 & 205 & 206 & 219 \\
\hline & \multirow{2}{*}{27} & Energy storage Capacity/kW·h & 0 & 0 & 417 & 417 & 417 & 417 \\
\hline & & Energy storage power/kW & 0 & 0 & 78 & 88 & 117 & 96 \\
\hline & \multirow{2}{*}{26} & Energy storage Capacity $/ \mathrm{kW} \cdot \mathrm{h}$ & 0 & 0 & 0 & 256 & 256 & 276 \\
\hline & & Energy storage power/kW & 0 & 0 & 0 & 27 & 27 & 26 \\
\hline & \multirow{2}{*}{6} & Energy storage Capacity/kW·h & 0 & 0 & 0 & 0 & 246 & 271 \\
\hline & & Energy storage power/kW & 0 & 0 & 0 & 0 & 27 & 27 \\
\hline & \multirow{2}{*}{28} & Energy storage Capacity $/ \mathrm{kW} \cdot \mathrm{h}$ & 0 & 0 & 0 & 0 & 0 & 711 \\
\hline & & Energy storage power/kW & 0 & 0 & 0 & 0 & 0 & 126 \\
\hline
\end{tabular}

Table 4. Result of four indexes in each scheme.

\begin{tabular}{ccccc}
\hline Schemes & $\begin{array}{c}\text { Total Energy Storage } \\
\text { Capacity/MW·h }\end{array}$ & $\begin{array}{c}\text { Annual Total } \\
\text { Abandoned PV } \\
\text { Power/MW }\end{array}$ & $\begin{array}{c}\text { The Average } \\
\text { Improvement of Node } \\
\text { Voltage Fluctuation/\% }\end{array}$ & $\begin{array}{c}\text { New PV } \\
\text { Accommodation } \\
\text { Capacity/MW }\end{array}$ \\
\hline 1 & 3.90 & 3.70 & 11.00 & 6.67 \\
2 & 6.06 & 3.09 & 13.89 & 7.09 \\
3 & 7.09 & 2.97 & 11.10 & 7.29 \\
4 & 7.27 & 2.81 & 9.75 & 7.39 \\
5 & 7.63 & 2.37 & 9.08 & 7.52 \\
6 & 8.38 & 2.02 & 9.87 & 7.72 \\
\hline
\end{tabular}

Since the PV power generated in the whole year is $8800 \mathrm{MW}$, the abandoned PV power in the results is in line with the requirements.

In Tables 3 and 4, a single node is for the energy storage configuration, which is the relative smallest in all schemes, but the annual total abandoned PV power in this scheme is the highest. Generally, the sum of energy storage and abandoned PV power is the lowest in all schemes. Since a single node cannot cooperate with other nodes, its new maximum accommodation ability is lowest under the determined energy storage capacity and annual abandoned PV power in scheme 1 . While the energy storage capacity is configured at six nodes in the sixth scheme, the new accommodation capacity is improved to $7.72 \mathrm{MW}$, the highest maximum accommodation capacity in all schemes. In the sixth scheme, the total energy storage configuration capacity is the highest, and the annual abandoned PV power is the lowest. However, the sum of energy storage and abandoned PV power is the largest. Different schemes have different energy storage capacities and abandoned PV power values. The maximum accommodation capacity is related to the number of the nodes and the sum of annual PV power and energy storage capacity. The average improvement indicator of node voltage fluctuation is not directly related to the number of node configurations. In general, the node voltage fluctuation was significantly improved due to the adjustment of energy storage and abandoned PV power. 


\subsection{Multi-Index Evaluation}

AHP and GRA are used for the comprehensive evaluation. In this paper, the important order of using AHP to determine weight is energy storage capacity, maximum accommodation capacity, abandoned PV power and average node voltage improvement level. It is verified that the consistency ratio (CR) in AHP is 0.0191, which passes the consistency test. The best resolution coefficient value $\rho$ is 0.3523 in this article. The results are shown in Table 5.

Table 5. Comprehensive results of multi-index evaluation.

\begin{tabular}{ccccccc}
\hline Scheme & $\mathbf{1}$ & $\mathbf{2}$ & $\mathbf{3}$ & $\mathbf{4}$ & $\mathbf{5}$ & $\mathbf{6}$ \\
\hline Weighted relevance & 0.6650 & 0.6226 & 0.6027 & 0.6182 & 0.6712 & 0.7979 \\
\hline
\end{tabular}

Scheme 6 has the highest weighted relevance with the optimized reference consequence. Since scheme 6 has more energy storage capacity configurations, coordinated work among nodes can increase the power consumption capacity of the distribution network. When the PV required by the distribution network needs to be further expanded, an additional expansion of $1.72 \mathrm{MW}$ of PV capacity can be carried out.

\section{Conclusions}

This article focuses on the evaluation and method of PV accommodation. The PSO-Monte Carlo algorithm is used to evaluate the distribution network's accommodation capacity. The staged control strategy of inverters and energy storage is used to ensure the safety and stability of the distribution system. The conclusions are as follows:

1. The PSO-Monte Carlo algorithm overcomes the shortcomings of the traditional PSO and Monte Carlo algorithms. The photovoltaic accommodation capacity assessment of the distribution network was improved in terms of the calculation speed and accuracy.

2. The siting of the energy storage is selected by the indictors of the degree to which the node voltage exceeds the limit and cluster strategy to avoid the randomness of addressing the bi-level planned algorithm.

3. According to the energy storage siting scheme, a cooperative game is used to optimize the annual energy storage and abandoned PV power of a node. This game improves the new accommodation capacity and the average degree of node voltage fluctuation.

4. Combined with the consideration of the actual situation, the introduction of two indicators, the average node voltage improvement and the limit accommodation capacity can more objectively reflect the impact of energy storage and abandoned photovoltaic on a distribution network. The final decision maker can make final decisions based on the importance of different indicators.

Author Contributions: J.G. and F.M. analyzed the data and wrote the paper; J.L. (Jixiang Lu) and J.L. (Jinjun Lu) performed the simulation and modeling; J.C. analyzed the results and reviewed the modeling and text; X.Z. prepared the data; L.L. contributed analysis tools. All authors have read and agreed to the published version of the manuscript.

Funding: This research was funded by State Key Laboratory of Smart Grid Protection and Control, SKL of SGPC, and National Key R\&D Program of China, grant number 2018YFB0905000, SGTJDK00DWJS1800232.

Conflicts of Interest: The authors declare no conflict of interest.

\section{References}

1. Khatib, H. Renewable energy in developing countries. In Proceedings of the International Conference on Renewable Energy-Clean Power 2001, IET, London, UK, 17-19 November 1993.

2. Al-Sumaiti, A.; Salama, M.M.A. Review on issues related to electric energy demand in distribution system for developing countries. In Proceedings of the 3rd IET International Conference on Clean Energy and Technology, Kuching, Sarawak, Malaysia, 24-26 November 2014. 
3. Wang, Q.; Hodge, B.M.S. Enhancing power system operational flexibility with flexible ramping products: A review. IEEE Trans. Ind. Inform. 2016, 13, 1652-1664. [CrossRef]

4. Amjad, A.M.; Alireza, S.; Taher, N. Multi-objective operation management of a renewable MG (micro-grid) with back-up micro-turbine fuel cell battery hybrid power source. Energy 2011, 36, 6490-6507.

5. Choi, J.H.; Kim, J.C. Network reconfiguration at the power distribution system with dispersed generations for loss reduction. In Proceedings of the IEEE Power Engineering Society Winter Meeting, Singapore, 23-27 January 2000.

6. Haque, M.M.; Wolfs, P. A review of high PV penetrations in LV distribution networks: Present status, impacts and mitigation measures. Renew. Sustain. Energy Rev. 2016, 62, 1195-1208. [CrossRef]

7. Teleke, S.; Jahanbakhsh, F.; Katiraei, F.; Agüero, J.R. Analysis of interconnection of photovoltaic distributed generation. In Proceedings of the 2011 IEEE Industry Applications Society Annual Meeting, Orlando, FL, USA, 9-13 October 2011; pp. 1-6.

8. Mamaghani, A.H.; Escandon, S.A.A. Techno-economic feasibility of photovoltaic, wind, diesel and hybrid electrification systems for off-grid rural electrification in Colombia. Renew. Energy 2016, 97, 293-305. [CrossRef]

9. El-Shimy, M.; Sharaf, A. Reduced-order modelling of solar-PV generators for small-signal stability assessment of power systems and estimation of maximum penetration levels. IET Gener. Transm. Distrib. 2018, 12, 1838-1847. [CrossRef]

10. Hoke, A.; Butler, R.; Hambrick, J. Steady-state analysis of maximum photovoltaic penetration levels on typical distribution feeders. IEEE Trans. Sustain. Energy 2013, 4, 350-357. [CrossRef]

11. Rylander, M.; Smith, J. Streamlined method for determining distribution system hosting capacity. IEEE Trans. Ind. Appl. 2016, 52, 105-111. [CrossRef]

12. Zio, E.; Delfanti, M. Monte Carlo simulation-based probabilistic assessment of DG penetration in medium voltage distribution networks. Int. J. Elec. Power 2015, 64, 852-860. [CrossRef]

13. Borges, R.T.; Salles, D.; Pereira, C.O. A comprehensive assessment of PV hosting capacity on low voltage distribution systems. IEEE Trans. Power Deliv. 2018, 33, 1002-1012.

14. Ayres, H.M.; Freitas, W. Method for determining the maximum allowable penetration level of distributed generation without steady-state voltage violations. IET Gener. Transm. Distrib. 2010, 4, 495-508. [CrossRef]

15. Koutroumpezis, G.N.; Safigianni, A.S. Optimum allocation of the maximum possible distributed generation penetration in a distribution network. Proc. CSUEPSA 2010, 80, 1421-1427. [CrossRef]

16. Ding, F.; Mather, B. On distributed PV hosting capacity estimation, sensitivity study and improvement. IEEE Trans. Sustain. Energy 2017, 8, 1010-1020. [CrossRef]

17. Giannitrapani, A.; Paoletti, S. Optimal allocation of energy storage systems for voltage control in LV distribution networks. IEEE Trans. Smart Grid. 2017, 8, 2859-2870. [CrossRef]

18. Zhao, B.; Xu, Z. Network partition-based zonal voltage control for distribution networks with distributed PV systems. IEEE Trans. Smart Grid. 2018, 9, 4087-4098. [CrossRef]

19. Capitanescu, F.; Ochoa, L.F. Assessing the potential of network reconfiguration to improve distributed generation hosting capacity in active distribution systems. IEEE Trans. Power Syst. 2015, 30, 346-356. [CrossRef]

20. Hashemi, S.; Ostergaard, J. Efficient control of active transformers for increasing the PV hosting capacity of LV grids. IEEE Trans. Ind. Inform. 2017, 13, 270-277. [CrossRef]

21. Divshali, P.H.; Soder, L. Improving hosting capacity of rooftop PVs by quadratic control of an LV-central BSS. IEEE Trans. Smart Grid. 2019, 10, 919-927. [CrossRef]

22. Jayasekara, N.; Masoum, M.A.S. Optimal operation of distributed energy storage systems to improve distribution network load and generation hosting capability. IEEE Trans. Sustain. Energy 2016, 7, 250-261. [CrossRef]

23. Wang, S.; Chen, S.; Ge, L. Distributed generation hosting capacity evaluation for distribution systems considering the robust optimal operation of OLTC and SVC. IEEE Trans. Sustain. Energy 2016, 7, 1111-1123. [CrossRef]

24. Chen, X.; Wu, W.; Zhang, B. Robust capacity assessment of distributed generation in unbalanced distribution networks incorporating ANM techniques. IEEE Trans. Sustain. Energy 2018, 9, 651-663. [CrossRef]

25. Shayani, R.A.; De Oliveira, M.A.G. Photovoltaic generation penetration limits in radial distribution systems. IEEE Trans. Power Syst. 2011, 26, 1625-1632. [CrossRef] 
26. Fu, Y.Y.; Chiang, H.D. Toward optimal multi-period network reconfiguration for increasing the hosting capacity of distribution networks. IEEE Trans. Power Deliv. 2018, 33, 2294-2304. [CrossRef]

27. Yao, H.; Du, X. Simulation of consumption capacity and voltage control strategy of distribution network with high penetration of photovoltaics. Power Syst. Tech. 2019, 43, 462-469.

28. Zhao, B.; Wei, L. Photovoltaic accommodation capacity determination of actual feeder based on stochastic scenarios analysis with storage system condidered. Auto Electric. Power Syst. 2015, 39, 34-40.

29. Tao, Q.; Sang, B. Optimized configuration method of distributed energy storage system in high photovoltaic penetration distribution network. High Volt. Eng. 2016, 42, 2158-2165.

30. Ding, M.; Fang, H. Optimal siting and sizing of distributed pv-storage in distribution network based on cluster partition. Proc. CSEE 2019, 39, 2187-2203.

31. Xue, L.; Jing, T. Boundary simulation of PV accommodation capacity of distribution network and comprehensive selection of accommodation scheme. Power Syst. Tech. 2020, 44, 907-916.

32. Wang, L.; Gao, M. Comprehensive evaluation of steganography analysis algorithm based on CRITIC weight and grey relation. Comput. Eng. 2017, 43, 154-159.

(C) 2020 by the authors. Licensee MDPI, Basel, Switzerland. This article is an open access article distributed under the terms and conditions of the Creative Commons Attribution (CC BY) license (http://creativecommons.org/licenses/by/4.0/). 